\title{
EL ANÁLISIS ORGANIZACIONAL Y DE POLÍTICA PÚBLICA A PARTIR DEL ENFOQUE DE REDES ${ }^{1}$
}

\section{ORGANIZATIONAL AND PUBLIC POLICY ANALYSIS FROM THE APPROACH OF NETWORKS}

\author{
ANÁLISE ORGANIZACIONAL E POLÍTICA PUBLICADA A PARTIR DA \\ ABORDAGEM DE REDE
}

\section{Por: TABARQUINO MUÑOZ - Raúl Andrés}

Candidato a PhD. en Administración, Magíster en Políticas Públicas y Economista de la Universidad del Valle; Investigador del Grupo “Gestión y Políticas Públicas” de la Universidad del Valle; Docente Tiempo Completo de la Universidad del Valle; E-mail: raul.tabarquino@correounivalle. edu.co - Colombia

\begin{abstract}
RESUMEN
El presente artículo indaga acerca de la rigurosidad del análisis organizacional y de política pública a partir del enfoque de redes, para ello se plantean dos casos el primero de orden de política pública en cuanto a los actores involucrados en el sector de las Tecnologías de la Información y las Comunicaciones (Tic) en Colombia y el segundo de orden organizacional la Cadena de Abastecimiento de la ESE Oriente de Cali, estos dos casos afianza que el Estado ya no es el actor principal en el accionar de la política pública y la dinámica organizacional de dichos sectores, sino por el contrario existen asociaciones público-privadas que coordinan e incentivan el desarrollo de infraestructura y consumo en un área determinada.
\end{abstract}

Palabras claves: Ciencia de las redes; Actores; Red.

JEL: C8; Z13

1. Este artículo es producto del proyecto de investigación adelantado por el autor para optar al título de Doctor en Administración de la Universidad del Valle, en particular en los ejes teórico y metodológico del enfoque de redes y a su vez de los conocimientos adquiridos en el curso de Ucinet 6.0 (programa de análisis de redes sociales) impartido por el organismo técnico de Capacitación ARS Chile entre los meses de septiembre y octubre de 2015. El proyecto doctoral del autor, se considera como un producto resultante de la formación de recurso humano en el marco del proyecto de investigación 8111, aprobado en la Convocatoria Interna 2013 de la Universidad del Valle y culminado en septiembre de 2015 por el grupo de Investigación en Políticas Públicas y Gestión. 


\begin{abstract}
This article asks about the thoroughness of organizational analysis and public policy from network approach to this, two cases arise the first order of public policy regarding the actors involved in the Information Technology sector and communications technology (Tic) in Colombia and the second of organizational order Supply Chain of ESE East Cali , these two cases strengthens the State is no longer the main actor in the actions of public policy and organizational dynamics of these sectors, but on the contrary there are public-private partnerships that coordinate and encourage infrastructure development and consumption in a given area.
\end{abstract}

Keywords: Network science; Actors; Net.

JEL: C8; Z13

\title{
RESUMO
}

ste artigo pergunta sobre o rigor da análise organizacional e as políticas públicas de estratégia de rede para isso, dois casos surgem a primeira ordem de políticas públicas Em relação aos atores envolvidos na indústria e tecnologia de comunicações de Tecnologia da Informação (TIC) na Colômbia e a segunda de ordem organizacional Supply Chain da ESE Médio Cali, Estes dois casos Reforça o Estado não é mais o principal jogador nas ações de políticas públicas e dinâmica organizacional desses setores, mas, pelo contrário, existem parcerias público -privadas que coordenam e incentivar o desenvolvimento de infra-estrutura e consumo dada na área.

Palavras-chave: Ciência da rede; atores; rede.

JEL: C8; Z13 


\section{INTRODUCCIÓN}

El análisis organizacional y de política pública son disciplinas de las ciencias administrativas y económicas porque en el ámbito político-estatal y social-humano, es necesario el debate y articulación de principios, derechos y procesos, que validen la importancia del entorno y actores en la consecución bienestar y avance discrecional de la sociedad, deconstruyendo acciones de política pública e interacciones interorganizacionales basadas en la dinámica de la oferta y demanda o en la dinámica de acciones racionales centradas en intereses colectivos o creados, con el establecimiento de marcos de la acción regulatoria que garanticen el proceder en la construcción de estrategias para el fortalecimiento de la estructura social a nivel individual o colectivo. Estos virajes conceptuales permiten indagar y profundizar un enfoque que reconozca la metamorfosis del entorno, la participación colectiva, la provisión y la heterogeneidad de actores que es más que simple pluralidad o social democracia, es el reconocimiento de la exploración de los espacios de complicidad entre Estado, el mercado y la sociedad (redes). Se trata de indagar ¿Cuál es la rigurosidad en el análisis organizacional y de política pública a partir del enfoque de redes?

\section{FUNDAMENTO TEÓRICO}

\subsection{La Ciencia de las Redes}

La ciencia de las redes (Network Science) nace de interacción entre la teoría de grafos, la física estadística y la dinámica no lineal (Tabarquino, 2015; Molina, 2004); el instrumento teórico y metodológico que incorpora esta ciencia se denomina análisis de redes sociales (Social Network Analysis), el cual, permite analizar un fenómeno, en la acción de las relaciones entre actores e intereses creados o naturales propios de la dinámica de interacción social de orden bilateral o multilateral llamada red social, que en últimas son el conjunto de relaciones sociales e interpersonales que ligan actores y organizaciones definidas en grupos (Tabarquino, 2015; Garrido, 2001; Sanz, 2003; Molina, 2004). El origen del análisis de redes sociales, se encuentra en el núcleo de las ciencias duras, específicamente en la matemática, en lo relacionado con el denominado problema histórico de los siete (7) puentes de Könisberg sobre el río Pregel de la ciudad prusiana de Kaliningrado, que es resuelto por el matemático suizo Leonhard Euler² (Perianes et al., 2008: 665); Posteriormente se avanza en la consolidación metodológica del análisis de redes sociales, con los desarrollos de la antropología social de Moreno (1934), el cual, aporta en relación a patrones de conexiones sociales que generan enlaces en conjuntos de actores.

Tabarquino (2015: 225-226), esboza las tres perspectivas teóricas del análisis de redes sociales: la primera corriente “...la antropología británica desarrollada a partir de las investigaciones del Instituto Rhodes-Livingston o Escuela de Manchester; donde se trabaja los conceptos de red, la intervinculación ("connectedness"), hoy

2. “...una serie de siete puentes unía una isla en el centro de la ciudad rodeada por el río Pregel con las dos márgenes del mismo. Se planteaba si era posible cruzar todos los puentes de manera que se pasara sólo una vez por cada uno de ellos” (Perianes, 2008: 5). 
reconceptualizado como densidad y el tratamiento sistemático en el libro Social networks in urban situations"; La segunda corriente “...la difusión de las investigaciones de la antropología británica y la tendencia norteamericana hacia los análisis cuantitativos y estadísticos, contribuyeron a expandir en Norteamérica la utilización y perfeccionamiento de la teoría/metodología del análisis de redes y ampliaron el ámbito de las investigaciones. El interés por la forma de las estructuras indujo al uso de la sociometría y propició el manejo de la teoría de grafos. La aplicación de estos métodos a poblaciones amplias y a un mayor número de relaciones hizo necesario el uso de matrices, que se vio favorecido por la aparición de los ordenadores personales. Los estudios efectuados por esta corriente de investigación, cuya contribución ha sido fundamental para el avance del análisis de redes, se han realizado casi siempre con la técnica de encuesta, preguntando sobre la composición, patrón de relaciones y contenidos de las redes del sujeto o "ego". Y la tercera corriente "...principalmente canadiense y norteamericana- es aquella que se ha ocupado de los procesos políticos como relaciones de intercambio y/o de dependencia entre grupos y Estados. Dentro de ella, una línea de gran influencia teórica y de interés en el campo de la acción colectiva y los movimientos sociales lo constituye la teoría de movilización de recursos, que explica los procesos políticos enfatizando el papel de los patrones de relaciones entre grupos de interés y la importancia dispar de las relaciones directas e indirectas para la obtención individual y grupal de los recursos..."

Explicado el panorama de las raíces del análisis de redes sociales (ARS), es ineludible definir el concepto de red como:

- "El conjunto de vínculos directos e indirectos definidos por relaciones mutuas de dependencia” (Scharpf, 1978: 362).

- Las redes son como un "complejo de organizaciones conectadas a las demás a través de dependencias de recursos y distinguidas de las otras por rupturas en las estructuras de dependencia de recursos” (Klijn, 1998: 33).

- "Las redes son el conjunto de relaciones relativamente estables, de naturaleza no jerárquica e interdependiente que vinculan a una variedad de organizaciones públicas, privadas y sociales que comparten intereses comunes y que intercambian recursos para perseguir esos intereses compartidos, admitiendo que la cooperación es la mejor manera de alcanzar las metas comunes” (Börzel, 1997: 2).

- "Totalidad de todas las unidades que están conectadas por un cierto tipo de relación” (Klijn, 1998: 33).

- “...es una estructura configurada por los vínculos, más o menos estables, que mantienen un determinado número de actores, públicos y privados, que intercambian recursos, materiales e inmateriales, en razón de su mutua dependencia en el proceso de formulación, decisión y ejecución de la políticas públicas dentro de un determinado ámbito sectorial o subsectorial” (Porras, 2001: 5).

- "Una estructura de interconexión inestable, compuesta por elementos de interacción, y cuya variabilidad obedece a alguna regla de funcionamiento" (Musso, 2004: 31). 
- "Las redes están formadas por entidades y por relaciones entre esas entidades; una relación es un conjunto de lazos de relacionamiento entre entidades, y las entidades que poseen un número de lazos de relacionamiento mayor a 1 son llamadas nodos. Las entidades y las relaciones apenas forman redes cuando posibilitan la existencia o son demandadas por el otro; o sea, un elemento no puede ser considerado un nodo a no ser que haya articulaciones con otros nodos; y él deja de serlo cuando esas articulaciones se acaban" (Kauchakje et al., 2006: 2).

- “... se entiende como un grupo de individuos que, en forma agrupada o individual, se relacionan con otros con un fin específico ... las redes pueden tener muchos o pocos actores y una o más clases de relaciones entre pares de actores" (Velázquez y Aguilar, 2005: 3).

Por, lo cual, las redes cumplen las siguientes características (Scharpf, 1978; Marsh y Rhodes, 1992; Jordana, 1995; Börzel 1997; Klijn, 1998; Porras, 2001; Petrizzo, 2004; Musso, 2004; Velázquez y Aguilar, 2005; Zurbriggen, 2011; Subirats et al., 2010; Latour 2005; Law, 2007):

- Las redes existen debido a las interdependencias entre los actores.

- Las redes constan de una diversidad de actores, en donde cada uno de los cuales tiene sus propias metas.

- Las redes son relaciones de naturaleza más o menos duradera entre actores.

- Las relaciones en la red entre los actores se caracterizan por la existencia de flujos de información.

- La red se compone de tres elementos básicos: nodos o actores, vínculos o relaciones y flujos ${ }^{3}$.

\subsection{La Metodología de la Ciencia de las redes}

La metodología de la ciencia de las redes se consolida como instrumento y se operativiza, por medio del álgebra matricial y de los grafos; el análisis de las relaciones se representa en forma de matriz con la existencia de una relación valor de 1 y la no existencia de una relación con un valor de 0 (Lozares 2005; Molina, 2004; Quiroga, 2003; Sanz, 2003; Velázquez y Aguilar, 2005; Wasserman y Fauts, 1994; Borgatti y Boston, 2003; Tabarquino, 2015).

Los elementos básicos que definen una red son esencialmente los actores que establecen las relaciones entre sí, y estas relaciones; los primeros son representados por puntos en la red o nodos y los segundos por líneas; los actores se describen como nodos y sus relaciones como líneas entre pares de nodos.

3. Nodos o Actores: Personas o grupos de personas que se encuentran en relación a un objetivo común; Vínculo o relaciones: Lazos que existen entre dos o más nodos; Flujo: Dirección del Vínculo. 
Tabla 1. Matriz de Relaciones de Adyacencia e Incidencia

\begin{tabular}{|c|c|c|c|c|c|c|c|}
\hline \multirow{3}{*}{ Jim } & $\mathrm{Jim}$ & Jill & Jen & Joe & \multirow{7}{*}{$\begin{array}{l}\text { Jim - Jill } \\
\text { Jim - Jen } \\
\text { Jim - Joe } \\
\text { Jill - Jen } \\
\text { Jill - Joe } \\
\text { Jen - Joe }\end{array}$} & Friendship & Proximity \\
\hline & - & 1 & 0 & 1 & & 1 & 3 \\
\hline & & & & & & 0 & 9 \\
\hline \multirow{4}{*}{$\begin{array}{l}\text { Jill } \\
\text { Jen }\end{array}$} & 1 & - & 1 & 0 & & 1 & 2 \\
\hline & 0 & 1 & - & 1 & & 1 & 1 \\
\hline & & & & & & 0 & 15 \\
\hline & 1 & 0 & 1 & - & & 1 & 3 \\
\hline
\end{tabular}

Fuente: Borgatti y Boston, 2003, p. 17.

Figura 1. Ejemplo de un Grafo

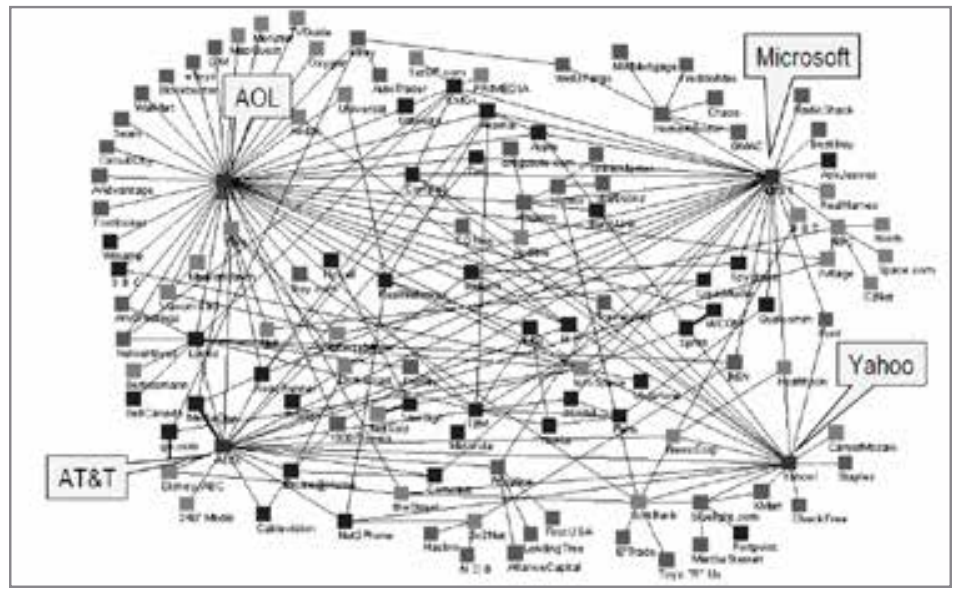

Fuente: Borgatti y Boston, 2003: 27.

\section{METODOLOGÍA}

Para lo concerniente a este artículo, la metodología empleada es mixta (Eisenhardt, 1989: 358; Patton, 2002) y se fundamenta en la utilización de fuentes cualitativas y cuantitativas de información. Se aplica el análisis de redes, utilizando el programa de redes (Ucinet 6.0), para comparar la red antes y después del diseño de la RISS. En el proceso metodológico, se trata de no ser subjetivo, por lo cual se combina los enfoques nominalista y realista de redes ${ }^{4}$ en el tratamiento de la in-

4. "El enfoque realista opta por delimitar las fronteras de la red en función de la percepción de los integrantes de la misma: son los propios integrantes quienes se reconocen como miembros de la red y, de esta forma, definen sus "fronteras". El enfoque nominalista, por el contrario, delimita las fronteras de la red en función de los intereses de la investigación, que establece cuáles son los límites de la población de objeto de estudio. Ambos enfoques pueden presentar problemas para el estudio de las redes de política: el enfoque realista puede incurrir en una sobre-estimación de las personas u organizaciones pertenecientes a una red, incluyendo actores irrelevantes o extrínsecos a la red; y, por su parte, la perspectiva nominalista tiende a restringir artificialmente el número de 
formación (cuadro de actores por su naturaleza y finalidad, Matriz de Adyacencia e incorporación de los datos en forma matricial en el programa de redes Ucinet 6.0). A continuación se describe los pasos del proceso metodológico, para efectuar el análisis de redes:

*Actores, Se describe el número de actores involucrados en la red, categorizando la naturaleza y el peso (Marsh y Rhodes, 1992; Jordana, 1995; Börzel 1997; Klijn, 1998; Porras, 2001; Petrizzo, 2004; Musso, 2004; Zurbriggen, 2011), con el fin de analizar detalladamente que actores representan la red, que necesariamente son el foco y el resto que no son el foco, pero tienen la capacidad de generar influencia.

* Matrices y visualización, de los diferentes actores en forma matricial (los actores estarán situados en las columnas, como si fueran variables, y a su vez en las filas, como si fuesen casos); la existencia de vínculo o relación entre estos el valor será uno (1) y si no existe un vínculo o relación el valor será cero (0).

*Análisis de la Estructura en $\operatorname{Red}^{\mathbf{5}}$ que se concentra principalmente en relaciones posibles, densidad, grado nodal (degree), indicadores descriptivos (media, desviación estándar e índice de centralización), grado de intermediación (betweenness), el grado de la Cercanía (closenness) si la matriz es simétrica y si no es simétrica se calcula el Power Bonicint.

\section{RESULTADOS Y DISCUSIÓN}

\subsection{Caso A. actores involucrados en sector de las Tecnologías de la Información y las Comunicaciones (Tic) en Colombia}

\section{A. Actores, Matriz de relaciones y Visualización de la Red}

Los actores involucrados en la política pública de Tic en Colombia son entes reguladores y operadores principales, distribuidos en dos grupos:

Tabla 2. Actores Involucrados en sector de las Tic en Colombia

\begin{tabular}{|l|c|}
\hline \multicolumn{1}{|c|}{ Entes Reguladores } & \multicolumn{1}{c|}{ Naturaleza } \\
\hline MINISTERIO DE TIC & Gubernamental \\
\hline CRC & Gubernamental \\
\hline ANE & Gubernamental \\
\hline SIC & Gubernamental \\
\hline
\end{tabular}

\begin{tabular}{|l|l|}
\hline $\begin{array}{c}\text { Operadores y prestadores } \\
\text { de Servicios }\end{array}$ & Naturaleza \\
\hline CLARO & Empresa Privada \\
\hline TELEFÓNICA-MOVISTAR & Empresa Privada \\
\hline TIGO & Empresa Privada \\
\hline UFF MÓVIL & Empresa Privada \\
\hline VIRGIN MOBILE & Empresa Privada \\
\hline ETB & Empresa Privada \\
\hline EDATEL UNE & Empresa Privada \\
\hline UNE EPM & Empresa Pública \\
\hline
\end{tabular}

Fuente: Tabarquino, 2015: 230.

miembros de la red y caer en el peligro de obviar a miembros y/o relaciones importantes (Howlett y Maragna, 2006: 434, citado por Rama y Luaces, 2007: 7, párr. 2).

5. Consultar los manuales de análisis de redes sociales de Velázquez y Aguilar, 2005; Quiroga, 2003. 
Posterior a la identificación de los actores, se correlacionan los diferentes actores en forma matricial, con el fin de visualizar y analizar la existencia o composición de la red de política pública. La valoración de la existencia de vinculo o relación entre estos el valor será uno (1) y si no existe un vínculo o relación el valor será cero (0). De manera siguiente, se procede a introducir los datos en el Programa de Unicet, con el fin de visualizar y analizar la estructura de la red de política pública.

Tabla 3. Matriz de Adyacencia de los actores de la política pública de Tic en Colombia

\begin{tabular}{|l|c|c|c|c|c|c|c|c|c|c|c|c|}
\hline & $\begin{array}{c}\text { MINI } \\
\text { DE TIC }\end{array}$ & CRC & ANE & SIC & CLARO & MOVISTAR & TIGO & $\begin{array}{c}\text { UFF } \\
\text { MÓVIL }\end{array}$ & $\begin{array}{c}\text { VIRGIN } \\
\text { MOBILE }\end{array}$ & ETB & $\begin{array}{c}\text { EDATEL } \\
\text { UNE }\end{array}$ & $\begin{array}{c}\text { UNE } \\
\text { EPM }\end{array}$ \\
\hline MINI DE TIC & 0 & 1 & 1 & 1 & 1 & 1 & 1 & 1 & 1 & 1 & 1 & 1 \\
\hline CRC & 1 & 0 & 1 & 1 & 1 & 1 & 1 & 1 & 1 & 1 & 1 & 1 \\
\hline ANE & 1 & 1 & 0 & 1 & 1 & 1 & 1 & 1 & 1 & 1 & 1 & 1 \\
\hline SIC & 1 & 1 & 1 & 0 & 1 & 1 & 1 & 1 & 1 & 1 & 1 & 1 \\
\hline CLARO & 1 & 1 & 1 & 1 & 0 & 1 & 1 & 0 & 0 & 1 & 1 & 1 \\
\hline MOVISTAR & 1 & 1 & 1 & 1 & 1 & 0 & 1 & 0 & 1 & 1 & 1 & 1 \\
\hline TIGO & 1 & 1 & 1 & 1 & 1 & 1 & 0 & 1 & 0 & 1 & 0 & 1 \\
\hline UFF MÓVIL & 1 & 1 & 1 & 1 & 0 & 0 & 1 & 0 & 0 & 0 & 0 & 0 \\
\hline VIRGIN MOBILE & 1 & 1 & 1 & 1 & 0 & 1 & 0 & 0 & 0 & 0 & 0 & 0 \\
\hline ETB & 1 & 1 & 1 & 1 & 1 & 1 & 1 & 0 & 0 & 0 & 1 & 1 \\
\hline EDATEL UNE & 1 & 1 & 1 & 1 & 1 & 1 & 0 & 0 & 0 & 1 & 0 & 1 \\
\hline UNE EPM & 1 & 1 & 1 & 1 & 1 & 1 & 1 & 0 & 0 & 1 & 1 & 0 \\
\hline
\end{tabular}

Figura 2. Grafo 1. Red de actores del sector de las Tic en Colombia Reguladores y principales operadores

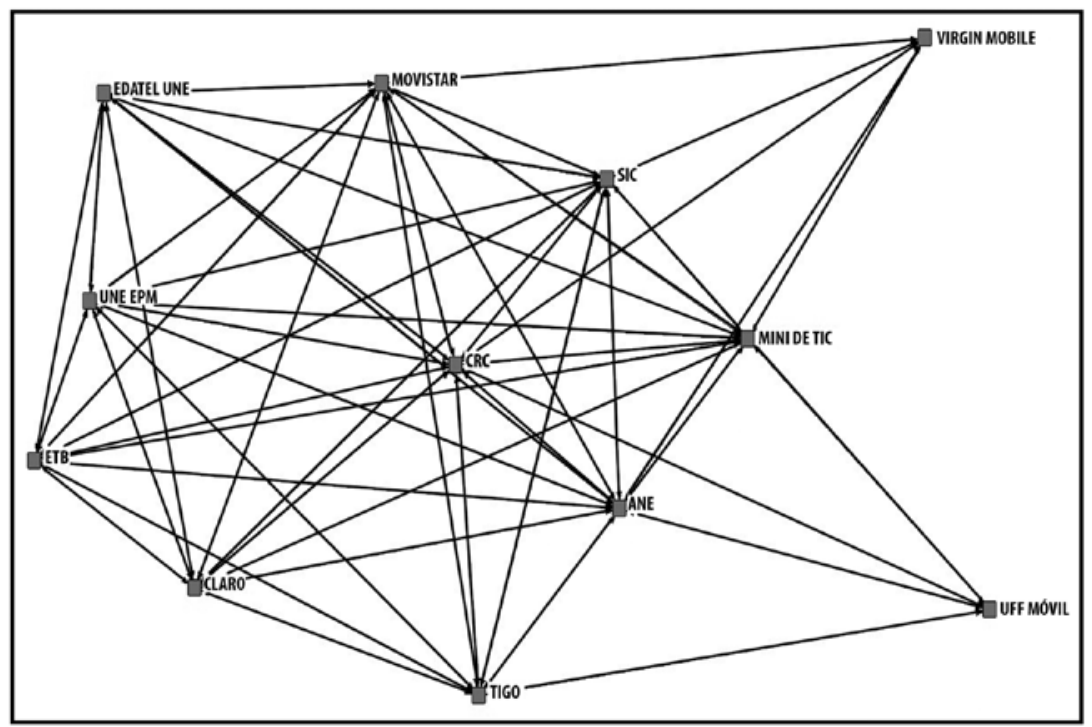

Fuente: Ucinet 6.0 
Tabla 4. B. Análisis de la Estructura en Red

\begin{tabular}{|c|c|}
\hline \multicolumn{2}{|c|}{ La Red de actores Involucrados en sector de las Tic en Colombia } \\
\hline $\begin{array}{l}\text { El total de las relaciones } \\
\text { posibles de la red }\end{array}$ & El total de las relaciones posibles de la red son de $132^{6}$ \\
\hline Densidad de la Red & $\begin{array}{l}\text { La densidad de la Red es del } 81,81 \%^{7} \text { - la conectividad entre } \\
\text { los actores de la red es alta- }\end{array}$ \\
\hline $\begin{array}{l}\text { Rango o El grado nodal } \\
\text { (degree) }\end{array}$ & $\begin{array}{l}\text { Los actores centrales de acuerdo al rango (Degree) son el Mi- } \\
\text { nisterio de Tic y la SIC por parte del Sector Gubernamental } \\
\text { (11) y Movistar por parte de Sector Privado (10), Une Epm por } \\
\text { parte de los operadores públicos (9) }\end{array}$ \\
\hline $\begin{array}{l}\text { La media de todas las rela- } \\
\text { ciones }\end{array}$ & $\begin{array}{l}\text { La media de todas las relaciones es } 9 \text {, lo cual, es un número } \\
\text { relevante de menciones en toda la red. }\end{array}$ \\
\hline La desviación estándar & $\begin{array}{l}\text { La media de todas las relaciones es } 9 \text {, lo cual, es un número } \\
\text { relevante de menciones en toda la red. }\end{array}$ \\
\hline $\begin{array}{l}\text { El Network Centralization } \\
\text { o índice de centralización }\end{array}$ & $\begin{array}{l}\text { La desviación estándar que es de } 2.041 \text {, implica una disper- } \\
\text { sión no considerable en la red entre los actores, lo cual, afir- } \\
\text { ma que la conectividad entre los actores de la red es alta. }\end{array}$ \\
\hline $\begin{array}{l}\text { El Grado de intermedia- } \\
\text { ción (betweenness) }\end{array}$ & $\begin{array}{l}\text { El actor de Mayor Intermediación en la red de política pú- } \\
\text { blica, es el Ministerio de Tic ( } 2.425) \text {, es decir, que es el nodo } \\
\text { central y el actor puente de la Red. }\end{array}$ \\
\hline $\begin{array}{l}\text { El grado de cercanía (clo- } \\
\text { senness) en la red se cal- } \\
\text { cula ya que la matriz es } \\
\text { simétrica }\end{array}$ & $\begin{array}{l}\text { El grado de cercanía en la red de política pública se calcula } \\
\text { ya que la matriz es simétrica, en el cual, el actor con mayor } \\
\text { grado de cercanía es el Ministerio de Tic (100). }\end{array}$ \\
\hline
\end{tabular}

\subsection{Caso B. La Cadena de Abastecimiento de la ESE Oriente de Cali}

El criterio para realizar el análisis de red de la ESE Oriente en la ciudad de Cali, se basa en el concepto de la cadena de abastecimiento, porque cumple con dos características: ${ }^{*}$ la primera, ser plataforma para el desarrollo de una red y * $\mathbf{l a}$ segunda, por su relación directa con la prestación del servicio (la satisfacción del buen servicio al usuario). No se aplica la parte administrativa como criterio, en relación a que solamente permite denotar las relaciones entre los funcionarios y no permite ver el flujo de los suministros entre los actores de la red.

6. Se calcula multiplicando el número total de nodos por el número total de nodos menos uno 12 $\mathrm{x}(12-1)=132$.

7. Se calcula dividiendo el número de relaciones existentes entre las posibles y multiplicado por 100. $108 / 132 \times 100=81,81 \%$. 


\section{A. Actores, Matriz de relaciones y Visualización de la Red $^{8}$}

Los diferentes actores se organizan en forma matricial de modo 1 de 33x 33 (matriz cuadrada, simétrica) (actores estarán situados en las columnas, como si fueran variables, y a su vez en las filas, como si fuesen casos); la existencia de vínculo o relación entre estos el valor será uno (1) y si no existe un vínculo o relación el valor será cero (0).

\section{Tabla 5. Actores de la cadena de abastecimiento de la ESE Oriente de Cali}

\begin{tabular}{|c|c|c|c|}
\hline No & Nombre y Atributo & \begin{tabular}{|} 
Área de Influencia \\
dentro de la RED
\end{tabular} & $\begin{array}{l}\text { Dinámica de actores en la } \\
\text { cadena de abastecimiento }\end{array}$ \\
\hline 1 & Hospital Carlos Holmes Trujillo & $\begin{array}{l}\text { Comunas } \\
13,14,15 \text { y } 21\end{array}$ & $\begin{array}{l}\text { Se correlaciona solo con los } \\
\text { centros de Salud de manera } \\
\text { logística y administrativa en- } \\
\text { trega de suministros; no inte- } \\
\text { ractúa con ningún puesto de } \\
\text { salud de manera logística en } \\
\text { la entrega de suministros }\end{array}$ \\
\hline 2 & Centro de Salud El Diamante & \multirow{9}{*}{ Comuna 13} & \multirow{15}{*}{$\begin{array}{l}\text { Los puestos de salud no se } \\
\text { correlacionan entre ellos en la } \\
\text { distribución de suministros; } \\
\text { la interacción dentro de la red } \\
\text { solo la pueden hacer con los } \\
\text { centros de salud }\end{array}$} \\
\hline 3 & Puesto de Salud Calipso & & \\
\hline 4 & Puesto de Salud Comuneros II & & \\
\hline 5 & Puesto de Salud Los Lagos & & \\
\hline 6 & Puesto de Salud Ricardo Balcázar & & \\
\hline 7 & Puesto de Salud Charco Azul & & \\
\hline 8 & Puesto de Salud Ulpiano Lloreda & & \\
\hline 9 & Puesto de Salud EI Poblado II & & \\
\hline 10 & Puesto de Salud El Vergel & & \\
\hline 11 & Centro de Salud Marroquín Cauquita & \multirow{6}{*}{ Comuna 14} & \\
\hline 12 & Centro de Salud Manuela Beltrán & & \\
\hline 13 & Puesto de Salud Alirio Mora Beltrán & & \\
\hline 14 & Puesto de Salud Alfonso Bonilla Aragón & & \\
\hline 15 & Puesto de Salud Los Naranjos & & \\
\hline 16 & Puesto de Salud Intervenidas & & \\
\hline
\end{tabular}

8 El conjunto de las comunas que atiende la ESE Oriente conforman un área bruta equivalente a 3.003,49 hectáreas y su población para el año 2014 corresponde a 614736 personas, cifras que equivalen al 3,24\% de la superficie y al 26,22\% de la población total del municipio de Santiago de Cali. Una extensa red de establecimientos de salud que presta servicios de promoción, prevención, diagnóstico, tratamiento, gestión de enfermedades, rehabilitación y cuidados paliativos, al igual que integra los programas focalizados en enfermedades, riesgos y poblaciones específicas, los servicios de salud personales y los servicios de salud pública. 


\begin{tabular}{|c|c|c|c|}
\hline No & Nombre y Atributo & $\begin{array}{l}\text { Área de Influencia } \\
\text { dentro de la RED }\end{array}$ & $\begin{array}{l}\text { Dinámica de actores en la } \\
\text { cadena de abastecimiento }\end{array}$ \\
\hline 17 & Centro de Salud El Vallado & \multirow{5}{*}{ Comuna 15} & \multirow{9}{*}{$\begin{array}{l}\text { Los puestos de salud no se } \\
\text { correlacionan entre ellos } \\
\text { en la distribución de sumi- } \\
\text { nistros; la interacción den- } \\
\text { tro de la red solo la pueden } \\
\text { hacer con los centros de } \\
\text { salud }\end{array}$} \\
\hline 18 & Puesto de Salud Comuneros & & \\
\hline 19 & Puesto de Salud Ciudad Córdoba & & \\
\hline 20 & Puesto de Salud Mojica & & \\
\hline 21 & Puesto de Salud El Retiro & & \\
\hline 22 & Centro de Salud Decepaz & \multirow{4}{*}{ Comuna 21} & \\
\hline 23 & Centro de Salud Potrero Grande & & \\
\hline 24 & Puesto de Salud Pizamos & & \\
\hline 25 & Puesto de Salud de Navarro & & \\
\hline 26 & Proveedor Mayorista - Adolfo Allers & \multirow{8}{*}{\multicolumn{2}{|c|}{$\begin{array}{l}\text { Se correlacionan solo con el Hospital Carlos Hol- } \\
\text { mes Trujillo para proveer insumos y material mé- } \\
\text { dico o administrativo }\end{array}$}} \\
\hline 27 & Proveedor Mayorista - Droguería Alianza & & \\
\hline 28 & Proveedor Mayorista - Equipadora Médica & & \\
\hline 29 & Proveedor Mayorista - Roche & & \\
\hline 30 & Proveedor Mayorista - LaFrancol & & \\
\hline 31 & Proveedor Minorista - Aseo Buriticá & & \\
\hline 32 & Proveedor Minorista - Institucional & & \\
\hline 33 & Proveedor Minorista - Papelería Andina & & \\
\hline
\end{tabular}

Tabla 6. Matriz de Adyacencia de los actores de la red de la Cadena de abastecimiento de la ESE Oriente Cali

\begin{tabular}{|c|c|c|c|c|c|c|c|c|c|c|c|c|c|c|c|c|c|}
\hline & H. Carl... & C.sE... & P.s Cali... & P.s Co... & P.s Lo... & P.s Ric.... & P.s Ch... & P.s Ulp... & P.s 日... & P.s 日... & C.s M... & C.s Ma... & P.sAlit... & Ps. Allo... & P.sLos... & P.s Inte... & I \\
\hline H. Carlos Holmes Trujllo & 0 & 1 & 0 & 0 & 0 & 0 & 0 & 0 & 0 & 0 & 1 & 1 & 0 & 0 & 0 & 0 & \\
\hline C.sElDiamante & 1 & 0 & 1 & 1 & 1 & 1 & 1 & 1 & 1 & 1 & 0 & 0 & 0 & 0 & 0 & 0 & \\
\hline Ps Calpso & 0 & 1 & 0 & 0 & 0 & 0 & 0 & 0 & 0 & 0 & 0 & 0 & 0 & 0 & 0 & 0 & \\
\hline Ps Comuneros II & 0 & 1 & 0 & 0 & 0 & 0 & 0 & 0 & 0 & 0 & 0 & 0 & 0 & 0 & 0 & 0 & \\
\hline P.s Los Lagos & 0 & 1 & 0 & 0 & 0 & 0 & 0 & 0 & 0 & 0 & 0 & 0 & 0 & 0 & 0 & 0 & \\
\hline P.s Ricardo Balcázar & 0 & 1 & 0 & 0 & 0 & 0 & 0 & 0 & 0 & 0 & 0 & 0 & 0 & 0 & 0 & 0 & \\
\hline P.s Charco Azul & 0 & 1 & 0 & 0 & 0 & 0 & 0 & 0 & 0 & 0 & 0 & 0 & 0 & 0 & 0 & 0 & \\
\hline P.s Ulpiano Loreda & 0 & 1 & 0 & 0 & 0 & 0 & 0 & 0 & 0 & 0 & 0 & 0 & 0 & 0 & 0 & 0 & \\
\hline P.s E Poblado II & 0 & 1 & 0 & 0 & 0 & 0 & 0 & 0 & 0 & 0 & 0 & 0 & 0 & 0 & 0 & 0 & \\
\hline Ps E Vergel & 0 & 1 & 0 & 0 & 0 & 0 & 0 & 0 & 0 & 0 & 0 & 0 & 0 & 0 & 0 & 0 & \\
\hline C.s Marroquin Cauquita & 1 & 0 & 0 & 0 & 0 & 0 & 0 & 0 & 0 & 0 & 0 & 1 & 1 & 1 & 1 & 1 & \\
\hline C.s Manuela Beltrán & 1 & 0 & 0 & 0 & 0 & 0 & 0 & 0 & 0 & 0 & 1 & 0 & 1 & 1 & 1 & 1 & \\
\hline 1 & & III & & & & & & & & & & & & & & , & \\
\hline
\end{tabular}

Al observar el comportamiento de la red a partir de la cadena de abastecimiento, se evidencia que el actor principal es el Hospital Carlos Holmes Trujillo, por ser el encargado de realizar los pedidos de toda la red tanto a los mayoristas como a los minoristas, y a su vez los distribuye a los centros de salud y estos últimos se encargan de coordinar la distribución de los suministros a los puestos de salud de su área de influencia. 
Figura 3. Grafo 2. La red de la Cadena de abastecimiento de la ESE Oriente Cali

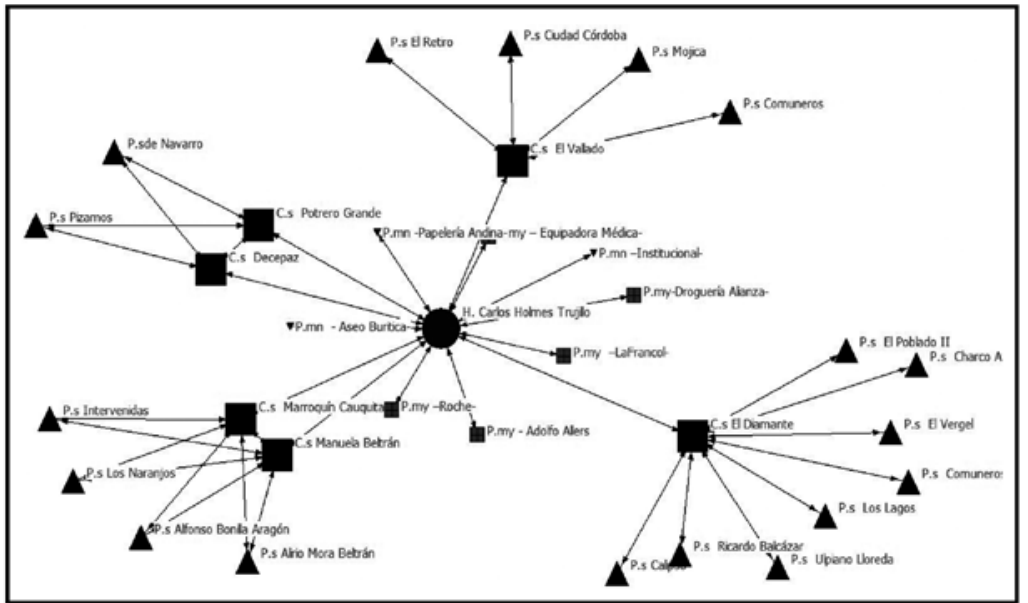

Fuente: Ucinet 6.0

Tabla 7. B. Análisis de la Estructura en Red

\section{Red de Cadena de Abastecimiento de la ESE Oriente de Cali}

\begin{tabular}{|l|l|}
\hline $\begin{array}{l}\text { El total de las relaciones posibles } \\
\text { de la red }\end{array}$ & 1056 \\
\hline Densidad de la Red ${ }^{10}$ & $\begin{array}{l}7,57 \% \text { - la conectividad entre los actores de la red es } \\
\text { baja- }\end{array}$ \\
\hline Rango o El grado nodal (degree) & El actor central es el hospital Carlos Holmes Trujillo (14) \\
\hline La media de todas las relaciones & $\begin{array}{l}2.4, \text { lo cual, no es un número relevante de menciones } \\
\text { en toda la red }\end{array}$ \\
\hline La desviación estándar & $\begin{array}{l}2.786, \text { lo cual, implica una dispersión no considerable } \\
\text { en la red entre los actores }\end{array}$ \\
\hline $\begin{array}{l}\text { El Network Centralization o índice } \\
\text { de centralización }\end{array}$ & $\begin{array}{l}38.51 \%, \text { esto indica que la red está cerca de no com- } \\
\text { portarse como una red estrella, en donde un actor jue- } \\
\text { ga un papel central que controla a toda la red }\end{array}$ \\
\hline $\begin{array}{l}\text { El Grado de intermediación (bet- } \\
\text { weenness) }\end{array}$ & $\begin{array}{l}\text { El actor de Mayor Intermediación en la red, es el Hospi- } \\
\text { tal Carlos Holmes Trujillo (429), es decir, que es el nodo } \\
\text { central y el actor puente de la Red }\end{array}$ \\
\hline $\begin{array}{l}\text { El grado de cercanía (closenness) } \\
\text { en la red se calcula ya que la ma- } \\
\text { triz es simétrica }\end{array}$ & $\begin{array}{l}\text { El actor con mayor grado de cercanía es el Hospital } \\
\text { Carlos Holmes Trujillo (64) }\end{array}$ \\
\hline
\end{tabular}

9. Se calcula multiplicando el número total de nodos por el número total de nodos menos uno $33 \mathrm{x}$ $(33-1)=1056$.

10. Se calcula de manera manual dividiendo el número de relaciones existentes entre las posibles y multiplicado por $100 \quad(80 / 1056 \times 100=7,57 \%)$ 


\section{CONCLUSIONES}

El enfoque de redes, se cimentó en los años 80, debido a la nueva lógica organizativa estructurada en las décadas de los 60 y 70, en cuanto a las trasformaciones tecnológicas, la globalización, el agotamiento del modelo fordista, la flexibilización del trabajo, la reinvención del gobierno -complejización y diversificación de la agenda pública-, la creciente movilización e influencia de los intereses privados y la fragmentación del Estado a raíz de la transferencia de competencias y recursos hacia nuevas entidades políticas (Porras, 2001: 721).

Más allá de estas definiciones o adecuaciones conceptuales, existen dos dimensiones, el análisis cuantitativo de redes como una tipología de intermediación de intereses y el análisis cualitativo de redes como una forma específica de gobernación; ambas dimensiones utilizan las redes como una herramienta analítica (Börzel 1997; Porras 2001; Petrizzo 2004); la primera considera el análisis de redes como un método del análisis de estructuras sociales; las relaciones entre los actores se estudian en términos de su cohesión, equivalencia estructural y representación espacial, utilizando métodos cuantitativos tales como clasificación jerárquica ascendente, tablas de densidad, block models; y la segunda, está más orientada hacia los procesos, se enfoca menos en la simple estructura de interacción entre actores y más en el contenido de esas interacciones utilizando métodos cualitativos tales como entrevistas en profundidad y análisis de discurso y contenido.

La principal contribución del enfoque de redes, es la elaboración de una concepción que trascienda la distinción tradicional entre agente y estructura, es decir, que ha desarrollado una concepción que trasciende las visiones sociocéntricas y Estado-céntricas. Como los sistemas no son observables directamente, entonces su estudio se hace por medio del examen y la observación de sus procesos internos, por lo cual, el análisis desde las redes para comprender las organizaciones y las políticas públicas como acciones, ofrece un medio para establecer un lazo entre las explicaciones de nivel micro y de nivel macro. Aunque el objetivo final no es sólo la explicación del comportamiento individual, esta aproximación considera que explicarlo o comprenderlo de manera suficiente es un paso intermedio y necesario para la explicación del funcionamiento del sistema político, ya que se considera que el comportamiento del sistema es la resultante de las acciones de sus partes componentes (Zurbriggen, 2011: 203). "Un concepto descentralizado de organización social y de gobernabilidad: La sociedad ya no está controlada por una inteligencia central (el Estado); más bien los mecanismos de control están dispersos y la inteligencia se distribuye entre una multiplicidad de actores (o unidades de procesamiento)” (Kenis y Schneider, 1991: 26, citado por Jordana, 1995: 83, Párr. 2).

La rigurosidad en el análisis organizacional y de política pública a partir del enfoque de redes permite denotar, describir e inferir cuales son los tejidos que se forman y se estructuran, sustentado en un juego dinámico donde existen preferencias de diferentes actores que reflejan en interdependencia o independencia si la categoría es el bien común; el objetivo del actor que era el principal (el Estado) es la inclusión social de la ciudadanía de un país, pero para los actores que antes eran de carácter irrelevante (operadores y proveedores) su finalidad es la consecución de la eficacia y eficiencia productiva de carácter de mejoramiento continuo en las aplicaciones de su valor de uso. 


\section{REFERENCIAS}

1. BÖRZEL, Tanja (1997). “Qué tienen de especial los policy networks? Explorando el concepto y su utilidad para el estudio de la gobernanza europea. Disponible en: http://www.unap.cl/p4_unap/docs/curso_sociologia/policy_networks.

2. BORGATTI, Steve y BOSTON, College (2003). Conceptos Básicos de Redes Sociales. Cancún (México).

3. EISENHARDT, K. M. (1989). Building Theories from Case Study Research. Academy of Management Review, 14(4), 532-550.

4. GARRID0, Francisco J. (2001). El análisis de redes en el desarrollo local. Facultad de Ciencias Políticas y Sociología, Universidad Complutense de Madrid.

5. JORDANA, Jacint (1995). El Análisis de los Policy Network ¿Una nueva perspectiva sobre la relación entre políticas públicas y Estado? Universidad Pompeu Fabra. Barcelona.

6. KAUCHAKJE, Samira; PENNA, Manoel Camillo; FREY, Klaus y DUARTE, Fabio (2006). Redes socio-técnicas y participación ciudadana: Propuestas conceptuales y analíticas para el uso de las Tics.

7. KLIJN, E. H. (1998). Redes de Políticas Públicas: una Visión General. Reimpresión y traducción por Mariángela Petrizzo con permiso de SAGE Publications Ltd.

8. LATOUR, B. (2005). Reassembling the Social: An introduction to Actor-Network-Theory. Oxford: Oxford University Press.

9. LAW, J. (2007). Actor Network Theory and Material Semiotics Centre for Science Studies, Lancaster University. Disponible en web: http://www. heterogeneities.net/publications/Law ANTandMaterialSemiotics.pdf>.

10. LOZARES, Carlos (2005). Bases socio-metodológicas para el Análisis de Redes Sociales, Empiria. Revista de Metodología de Ciencias Sociales, 10, julio-diciembre, pp. 9-35.

11. MARSH, D. Y RHODES, R.A.W. (eds.) (1992). Policy networks: Empirical evidence and theoretical considerations, Westview Oress, Boulder C0.

12. MOLINA, José Luis (2004). La ciencia de las redes, Apuntes de Ciencia y Tecnología 11, Junio (36-42).

13. MUSSO, P. (2004). A filosofía da rede. In: Parente, A. (org.) Tramas da rede: novas dimensões filosóficas, estéticas e políticas da comunicação. Porto Alegre: Sulina.

14. PERIANES R., ANTONI0; OLMEDA G., Carlos y DE MOYA A. Félix (2008). Introducción al Análisis de Redes. Revista. El profesional de la información, v. 17, n. 6, pp. 664-669.

15. PATTON, M.Q. (2002). Qualitative Research and Evaluation Methods. Thousand Oaks, CA: Sage.

16. PETRIZZO PÁEZ, Mariángela (2004). Redes e institucionalización: vinculando evidencias empíricas y redes políticas. Instituto Universitario Ortega y Gasset.

17. PORRAS MARTínEZ, José Ignacio (2001). Policy Network o Redes de Política Pública: Una Introducción a su Metodología de Investigación. Estudios Sociológicos XIX.

18. QUIROGA, Águeda (2003). Introducción al Análisis de Datos Reticulares Prácticas con UCINET6 y NetDraw1 Versión 1. Departamento de Ciencias Políticas, Universidad Pompeu Fabra.

19. RAMA, Paula y LUACES, Pilar (2007). Análisis de redes aplicado a la formación de la agenda pública en las conurbaciones gallegas: los casos de Vigo y La Coruña. IX Congreso de la Federación Española de Sociología, Barcelona.

20. SANZ MENÉNDEZ, Luis (2003). Análisis de redes sociales: o cómo representar las estructuras sociales subyacentes. Apuntes de Ciencia y Tecnología, 7, pp. 21-29.

21. SCHARPF, F.W. (1978). Interorganizational policy studies: issues, concepts and perspectives in K.I. Hanf y F.W. Scharpf (eds) pp. 345-70.

22. SUBIRATS, J.; GRAU, M.; ÍNIGUEZ-RUEDA, L. (2010) La Perspectiva Sociotécnica en el Análisis de Políticas Públicas. Universidad Autónoma de Barcelona (Versión Electrónica).

23. TABARQUINO MUÑOZ, Raúl Andrés (2015). La red de política pública de TIC en Colombia. Actores reguladores y principales operadores. Estudios Políticos, 46, Instituto de Estudios Políticos, Universidad de Antioquia, pp. 221-241.

24. VELÁZQUEZ ÁLVAREZ, Alejandro y AGUILAR GALLEGOS, Norman (2005). Manual Introductorio al Análisis de Redes Sociales.

25. WASSERMAN, Stanley y FAUST, Katherine (1994). Social Network Analysis: Methods and Applications. Cambridge: Cambridge University.

26. ZURBRIGGEN, Cristina (2011). La utilidad del Análisis de Redes de Políticas Públicas. Argumentos, vol. 24, núm. 66, may0-agosto, 2011, pp. 181-208 Universidad Autónoma Metropolitana. Distrito Federal, México. 